\title{
NK 细胞介导的脾脏细胞清除实验
}

\section{NK Cell Mediated Splenocyte Rejection Assay}

\author{
冯瑾*
}

医学院免疫所, 清华大学, 北京

*通讯作者邮箱: fengj17@mails.tsinghua.edu.cn

引用格式: 冯瑾 (2019). NK 细胞介导的脾脏细胞清除实验. Bio-101 e1010334. Doi: 10.21769/BioProtoc. 1010334.

How to cite: Feng, J. (2019). NK Cell Mediated Splenocyte Rejection Assay. Bio-101 e1010334. Doi: 10.21769/BioProtoc.1010334. (in Chinese)

摘要: $\beta 2 \mathrm{~m}$ 是 MHC-I 类分子重要的组成成分， $\beta 2 \mathrm{~m}$ 缺失的小鼠不表达 MHC-I 类分子。 $N K$ 细胞的主要功能是杀伤功能, 为了研究 $N K$ 细胞的功能, 我们注射 $\beta 2 m$ 缺失小鼠的 脾脏细胞至 NK 细胞功能正常的野生型小鼠, 功能正常的 NK 细胞能够清除 $\beta 2 m$ 缺失 的细胞, 体内清除 $\beta 2 m$ 缺失细胞的效率则可以反映 $N K$ 细胞的功能。

关键词: NK 细胞, 脾脏细胞清除, 体内功能

研究背景：固有免疫系统主要成员 NK 细胞能够杀死多种病毒感染细胞, 肿瘤细胞, 以 及参与异基因骨髓移植排斥反应而在免疫防御的过程中扮演着重要角色。NK 细胞表面 表达大量的活化性和抑制性受体，NK 细胞的功能状态取决于这两种受体信号的平衡。 目前的研究显示 NK 细胞以“丢失自我”和“诱导自我”两种模式识别异己，这两种识别方 式使得 NK 细胞打破了活化和抑制信号的平衡进而清除异源细胞。“丢失自我”的识别模 式认为正常细胞表面广泛表达 MHC-I 类分子, 它们能够被 NK 细胞表面的 MHC-I 类分 子依赖的抑制性受体所识别，这种识别介导了强的抑制信号的传递。NK 细胞接受的由 自身 MHC-I 类分子识别所传递的抑制信号强于 NK 细胞表面活化性受体识别所传递的 活化信号, 因此 NK 细胞不杀伤正常细胞。当靶细胞上的 MHC-I 类分子不匹配、表达下 降或者丢失时, $\mathrm{NK}$ 细胞上由 MHC-I 类分子介导的抑制信号被移除, NK 细胞表面活化 性受体介导的活化信号占主导地位，NK 细胞活化进而杀伤靶细胞。 


\section{材料与试剂}

1. 铌子

2. 剪刀

3. $60 \mathrm{~mm} \times 15 \mathrm{~mm}$ 平血 (Corning, catalog number: 430166)

4. $15 \mathrm{ml}$ 离心管 (BD Falcon, catalog number: 352096)

5. 100 目滤网

6. $1 \mathrm{ml}$ 注射器

7. 注射器活塞

8. 待检测小鼠, 一般每组 3 5 只

9. B6 野生型小鼠

10. $\beta 2 \mathrm{~m}$ 缺失小鼠

11. 胎牛血清

12. Poly I:C (InvivoGen, catalog number: 31852-29-6)

13. 红细胞裂解液 (eBioscience, catalog number: 00-4333-57)

14. PBS

15. 1640 培养基 (WISENT, catalog number: 350-006-CL)

16. CFSE (eBioscience, catalog number: 65-0850-84)

17. $10 \mu \mathrm{M}$ CFSE (见溶液配方)

18. $1 \mu \mathrm{M}$ CFSE (见溶液配方)

\section{仪器设备}

1. 细胞计数仪

2. 多色流式细胞仪

3. 超净工作台

4. 水浴锅

5. 离心机

\section{实验步骤}

1. 小鼠腹腔注射 $200 \mu \mathrm{l} 1 \mu \mathrm{g} / \mu \mathrm{l}$ 的 polyl:C, 18 24 小时后用于后续实验。 
2. 断颈处死小鼠, 在超净台从小鼠腹腔取 $\beta 2 \mathrm{~m} \mathrm{KO}$ 和 WT 小鼠脾脏, 放于含 100 目 笁网和 $3 \mathrm{ml}$ PBS 的 $60 \mathrm{~mm} \times 15 \mathrm{~mm}$ 平血中用注射器活塞研磨至无组织块, 收集 细胞至 $15 \mathrm{ml}$ 离心管。

3. $400 \times g$ 离心 $5 \mathrm{~min}$ 。

4. 弃上清, 加 $1 \mathrm{ml} 1 \mathrm{x}$ 红细胞裂解液, 混匀, 冰上裂解 $5 \mathrm{~min}$ 。

5. 裂解完成后可见液体红色变淡, 加 $5 \mathrm{ml} \mathrm{PBS}$ 终止反应, $400 \times \mathrm{g}$ 离心 $5 \mathrm{~min}$ 。

6. 用 $800 \mu \mathrm{l}$ PBS 重悬 $\beta 2 \mathrm{~m} \mathrm{KO}$ 和 WT 细胞沉淀, 取 $400 \mu \mathrm{l}$ 重悬的细胞进行实验。

7. CFSE 母液浓度为 $5 \mathrm{mM}$, 配制成 $1 \mu \mathrm{M}$ 和 $10 \mu \mathrm{M}$ 工作浓度, 避光操作 (见溶液配 方)。

8. 混匀后, $37^{\circ} \mathrm{C}$ 水浴锅 8 分钟, 每 2 分钟混匀一次, 避光操作。

9. 迅速加入 $800 \mu \mathrm{lFBS}$, 终止反应。

10. 加 $8 \mathrm{ml} \mathrm{PBS,} 400 \times \mathrm{g}$ 离心 $5 \mathrm{~min}$, 洗 2 次。

11. 使用流式细胞仪检测 CFSE 标记情况，并保存注射前流式图。

12. 两种细胞 $1: 1$ 混合, 尾静脉注射 $400 \mu \mathrm{l}$ (每种鼠 $2 \times 10^{6}$ 个细胞) 至提前注射了 polyl:C 的受体鼠。

13. 6 小时后, 使用流式细胞仪检测小鼠脾脏和淋巴结中 CFSE 阳性的细胞数, 如图 1 所示收取 $1 \times 10^{3}$ 的 CFSE low 的细胞, 计算出 CFSEhigh 的细胞的清除效率。清除效 率: $100 \times\left[1-\right.$ (收取的 CFSEhigh 细胞数目 $\times$ 注射时 CFSE ${ }^{\text {low }}$ 的细胞比例) $/($ 收取的 CFSElow 细胞数目 $\times$ 注射时 CFSEhigh 的细胞比例)。

\section{结果与分析}

分析结果见图 1 。 
A.

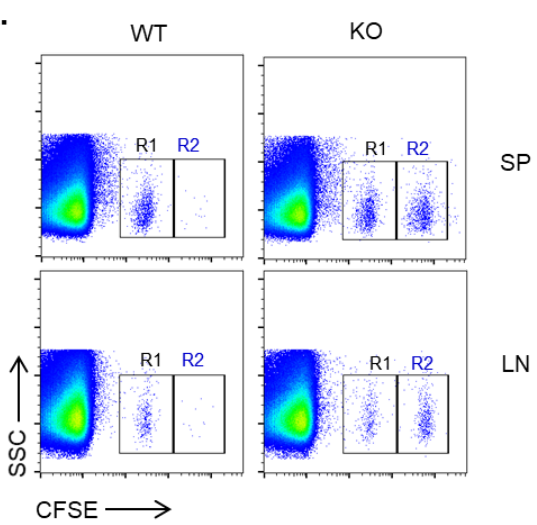

B.

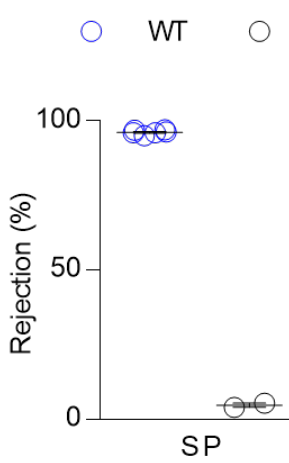

$\beta 2 \mathrm{~m} \mathrm{KO}$

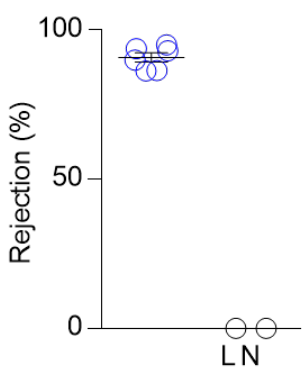

图 1. WT 和 KO 鼠脾细胞清除效率检测. A. 流式检测 WT 和 $K O$ 鼠脾细胞清除;

B. 统计分析 WT 和 KO 鼠脾细胞清除效率。

\section{溶液配方}

1. $10 \mu \mathrm{M}$ CFSE

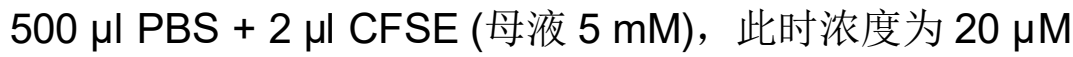

混匀, 取 $400 \mu \mathrm{l}$ 与 $400 \mu \mathrm{l}$ PBS $(\beta 2 \mathrm{~m} \mathrm{KO})$ 细胞液混匀

2. $1 \mu \mathrm{M}$ CFSE

$100 \mu \mathrm{l} 20 \mu \mathrm{M}$ CFSE $+900 \mu \mathrm{l} \mathrm{PBS}$, 此时浓度为 $2 \mu \mathrm{M}$

混匀，取 $400 \mu \mathrm{l}$ 与 $400 \mu \mathrm{l}$ PBS (WT) 细胞液混合

\section{致谢}

董忠军实验室的工作得到清华大学免疫所的支持。本文实验方案改编自本实验室发表 的文章 (Chen 等, 2016)。

\section{参考文献}

1. Chen, S., Yang, M., Du, J., Li, D., Li, Z., Cai, C., Ma, Y., Zhang, L., Tian, Z. and Dong, Z. (2016). The self-specific activation receptor SLAM family is critical for NK cell education. Immunity 45(2): 292-304. 